\title{
A Survey on Personalized Recommendation System for Web Services
}

\author{
Karishma Singh ${ }^{1}$, Analp Pathak ${ }^{2}$ \\ ${ }^{1}$ M.Tech Student, Department of computer Science, SRM University, Chennai, India \\ ${ }^{2}$ Assistant Professor, Department of computer Science, SRM University, Chennai, India
}

\begin{abstract}
Personalized web service recommendation systems. Web services are integrated software components for the support of interoperable machines interaction over a network, Many different Techniques and algorithms of recommendations are discussed, In this paper we present the recommendation system related most popular techniques with their pros and cons. Collaborative filtering technique is a most popular technique used for the recommendation system. We will present the research related to collaborative filtering has been done. In the end we will show the main challenges of recommender systems.
\end{abstract}

Keywords: Personalized, Recommendation, Web services, collaborative filtering, Issues

\section{Introduction}

The area of Personalized Recommendation System is becoming very popular because nowadays information is overloaded everywhere and in these situations Recommendation comes for the rescue. A lot of work has been done in this area in academic and industrial domains over the years. A Personalized recommendation system basically compares a user profile to some reference characteristics, and then predict the 'rating' or 'preference' that a user would give to an item they had not yet considered. The aim of a Recommendation System is to generate a set of meaningful recommendations for a set of users regarding products or some items in which they have interests. The design of recommendation systems depends on the domain of the system and the particular characteristics of the data available [1].

Web services are software components designed to support interoperable machine-to-machine interaction over a network. Web services are widely spreading by their interoperability, loose coupling, reusability and extensibility with the help of its components namely UDDI (Universal Description Discovery and Integration), WSDL (Web Service Description Language), XML (extended Markup Language), and SOAP (Simple Object Access Protocol). UDDI is a registry where service provider registers their services, WSDL is used for describing the services; SOAP is used to transfer the data, enables communication between service provider and service requester. XML uses custom defined tags to describe the data in a structured manner [3]. With the increase of number of web services it is difficult to find the best service. Quality of service (QoS) is used to represent the non functional requirements of web service. QoS is defined as set of properties like response time, availability and reputation etc. The values of the user independent QoS properties are usually offered by service providers or by third-party registries for e.g., UDDI. It is difficult to evaluate the each web service because of following reasons.
- It ee $^{\mathrm{s}}$ impossible for the user to check the QoS(Quality of service) value of all the services because it is a time consuming task to do.

- Some QoS Properties like reliability and reputation are difficult to be evaluated because they need the long observation time.

- Inappropriate service selection cause many problems to the resulting application.

Optimal Web service selection and recommendation are thus difficult to achieve. In this paper, In Section II we studied basics Recommendation technique, In Section III we presented research related to Personalized Recommendation System with their techniques and pros and cons, In section IV we presented the various issues related to Recommendation.

\section{Recommendation System}

Recommender systems are information filtering system that recommend products or services based on users past behavior or consumption patterns. Recommender system is broadly classified as Content-based, Knowledge-based, Collaborative Filtering and demographic-based [2] [3].

\subsection{Recommendation System Techniques}

\subsubsection{Collaborative Filtering}

Paul Resnick and Hal Varian [4] describes the idea of collaborative filtering is to find users in a community that share appreciations [5]. If two users have same or almost same rated items in common, then they are considered as similar taste user [6]. Such users build a group or a neighborhood. A user gets to those items that he has not rated before, but that were already positively rated by users in his neighborhood.

Drawbacks:- Suffer from gray sheep problem, cold-start problem, shilling attack, and Malicious users ${ }^{\text {ee }}$ rating. 


\section{International Journal of Science and Research (IJSR) \\ ISSN (Online): 2319-7064}

Index Copernicus Value (2013): 6.14 | Impact Factor (2014): 5.611

\subsubsection{Content based Filtering}

Content-based recommendation method is based on the information about item content and ratings a user has given to items. This technique combines these ratings to profile of the useres interests based on the features of the rated items. The recommendation engine then can find items with the preferred in the past as The recommendations of a contentbased system are based on individual information and ignore contributions from other users[7].

Drawbacks:- Incapable to find users ${ }^{\text {ee }}$ interest towards products or services Impossible to find product quality.

\subsubsection{Knowledge-based}

It Recommends products by considering the user History, then it predict the user preference based on the inferences.

Drawbacks:- User profile maintenance is a difficult work.

\subsubsection{Demographic-based Recommendation}

It recommends the product by considering the user's demographic data like gender, age, date of birth, educational qualification and other personal features.

Drawbacks:- There is a Possibility of wrong personal categorization.

\subsubsection{Hybrid Recommendation Systems}

All the above described systems have their strengths and weaknesses. A Hybrid recommendation System combines the two or more techniques to gain more strength and to reduce the weaknesses of individual technique by combining it with other technique. The most popular Hybrids are those of Content-based and Collaborative Filtering.

Drawbacks:- Inappropriate combination of recommender technique leads to poor recommendation or decrease precision result.

\subsection{Collaborative Filtering Method}

Breese et al. [1] divide the Collaborative Filtering algorithms into two broad classes: memory based algorithms and model based algorithms. Collaborative Filtering (CF) is widely employed in commercial recommender systems, such as and Amazon.com. The basic idea of CF is to predict and recommend potential favorite items for a particular user employing rating data collected from other users. CF is based on processing the user-item matrix.

\subsubsection{Model Based Collaborative Filtering}

Modez based collaborative filtering technique is a based on learned models. It analyze the training data, summarize the complicated patterns into the learned models, and then make predictions based on the models. It uses behavior to build of line models like Bayesian, that capture the relationship between the item or user relations. Matrix factorization is one of the most popular model-based CF approaches, which was first introduced to address the QoS prediction problem in [8]. Drawbacks:- Inflexible Quality of predictions , Synonyms, Problem and Cold-start problem.

\subsubsection{Memory Based Collaborative Filtering}

Memory based collaborative filtering techniques consider item to item or user to user similarity to make prediction for user on the items which user has not used yet. For prediction, whole training set is taken into memory, making it easier to include new data but it is slow in performance on large datasets. This issue can be overcome by Precalculating correlations and it. Memory based collaborating filtering technique are categorized into two types user based collaborative filtering and item based Collaborative filtering.

\section{a) Item based collaborative Filtering}

Item based Collaborative Filtering Recommends product or based on item-item similarity This technique first find the correlation between the users and then based on this correlation it predicts the unknown rating of the item for the user.

Drawbacks:- Data Sparsity, Scalability doesn't generalize data, Gray sheep problem , Cold-start problem Ramp-up problem is more stable than between users.

\section{b) User based collaborative Filtering}

User based Collaborative Filtering Recommends product or service based on user-user similarity. It recommendation systems, the prediction of the rating of an item for a given user is done depending upon the ratings for the same item rated by different similar users.

Drawback:- Data Sparsity, Scalability, Doesn't generalize data Gray sheep problem Cold-start problem Ramp-up problem.

\section{Related Research}

J.S. Breese, D. Heckerman, and C. Kadie [2] propose recommender systems usage a database about user given ratings to calculate subjects or goods a new user might similar. They have described another task which depends on correlation coefficients, vector-based same calculations, and arithmetical Bayesian methods. Collaborative filtering algorithm is used two classes:

(i) In Memory-based algorithms, work is to create expectation over the whole user database. Normally, this task is to predict the votes of a specific user from a database of user elections from a section by using the Collaborative Filtering. It is easy to implement and requires no training cost. It can easily take new users ${ }^{\text {ee }}$ ratings into account. Some drawbacks are. It cannot cope well with large data, since their online performance is often slow.

(ii) In Model-based collaborative filtering, it used to evaluate the user record, which is then used for calculations. this task can be observed as finding the predictable value of a rating, assumed what they know about the user. They request to expect rating on unnoticed items for the active user. It can quickly generate recommendations and can achieve good performance. The difficulties of these networks are. It has lesser memory requirements. 


\section{International Journal of Science and Research (IJSR) \\ ISSN (Online): 2319-7064}

Index Copernicus Value (2013): 6.14 | Impact Factor (2014): 5.611

Z. Maamar, S.K. Mostefaoui, and Q.H. Mahmoud [9] have worked on context. Web service personalization is developed by using the contexts. Context is the information, It characterizes the relationship among people, applications, and location. Web services are personalized so that users ${ }^{\text {ee }}$ can be owned. Preferences are dissimilar types. Preferences are measured based on performance of Web services when it starts and ends. Personalization has two types such as explicit or implicit. Straight participation of the users in the modification of applications can be used with explicit personalization. Users clearly define the data that need to be preserved or rejected. Implicit personalization does not call the some users participation and can be made upon learning plans that path users' behaviors and benefits. Personalization is based on the personal attributes of stationary user, mobile and locations. They have used some context such as Ucontext, W-context, and R-context. U-context is used to represent status of a user and returns his individual preferences in relations of execution location and implementation time of services. R-context is used to represent the status of a resource. W-context is used to represent status of a Web services and also the implementation constraints on the Web service. They have provided some policies like consistency, inspection and feasibility. Its advantages are the interaction of the web services is well based on context and It highlighted all the resources on which the web service performed.

X. Chen et al. [10] describes in their paper that effective QoS based recommendation is becoming more important and previous could not consider QoS variance according to the user's location and provided just the limited information on the performance of service candidates. Their paper proposes a collaborative filtering algorithm designed for large scale Web Services. The recommendation approach makes use of region-based Collaborative Filtering algorithm and consists of two phase process. In first phase, users are divided into different regions based on their physical location and previous QoS experience on Web Services. In the second phase, when a user is requesting Web Services, it finds similar users for the current user and makes predictions for Web Services which have the best predicted QoS values for the unused services.

J. Yin et al. [11] says that QoS values are very important and propose a novel collaborative QoS prediction framework. Suppose there are $\mathrm{i}$ users and $\mathrm{j}$ Web Services, and they contribute to an $\mathrm{i} x \mathrm{j}$ web service QoS matrix R, and each entry of rating $r$ represents a QoS value recording the specific usage information of web service $k$ executed by user a and predicts missing QoS values of Web Services by using the concept of localization and matrix factorization. This approach assumes that users nearby share similar web service invocation experience and makes of matrix factorization framework for predicting missing QoS values.

J. Zhu et al. [12] propose a novel clustering-based QoS prediction framework, in which various Landmarks (computers) are deployed in the internet to monitor QoS information of the available Web Services by invoking these services at regular intervals and then cluster the computers based on the QoS information that has been obtained. It then clusters these small groups into a large existing cluster, and try to form hierarchy of clusters, this is done by measuring the latency between the landmark and the cluster, from this QoS predictions are made from the QoS information that has been gained from the landmarks.

G. Kang et al. [13] propose a Web Services recommendation approach which recommends Web Services to a user based on the user's history. The system measures the similarity between the user's functional interests and web services and based on the similarity in the functional and non functional characteristics Active Web Service Recommendation System, ranks the services so that a list is generated which has top recommendations for the user.

Z. Zheng et al. [14] present a collaborative filtering approach for predicting QoS values of Web Services. It proposes a prototype called Web Service Recommendation (WSRec) which makes use of user-collaborative mechanism for collecting Web Services QoS information from different users, and based on the collected QoS information, predictions are made using the collaborative filtering approach. WSRec is a centralized server which consists of web service QoS data for various Web Services contributed by service users and makes recommendations for the user requesting a web service.

J.E. Haddad et al. [15] address the issue of recommending Web Services by considering into account transactional properties like compensable or not, QoS characteristics, and also the functional requirements of Web Services according to the requirements of the user. The web service composition can be viewed as a three stage process. This paper has focused on designing a composite web service by designing an algorithm which integrates QoS and transactional properties that will ensure proper execution. In this, mainly five QoS criteria like execution price, availability, reputation, successful execution rate, and execution duration have been used and selecting a local QoS-driven service related to these.

L. Shao et al. [16] propose that non-functional properties such as quality of service (QoS), should be taken into consideration when making recommendations to the customers. This paper makes use of the concept of similarity mining through collaborative filtering for making predictions to the users from other consumer experiences.

X. Dong, A. Halevy, J. Madhavan, E. Nemes, and J. Zhang [8] have presented the fundamental alogorithm the Woogle search engine for web services. It makes provisions for similarity search for web services, such as finding the similar web-service procedures. It is determining procedures that are combining with a certain one. They have described novel techniques to funding these types of searches. The main contribution of this work has a simple group of search functionalities that a web-service search engine should be provision. They have clustered several sources of suggestion in order and also to decide similarity among web-service procedures. The main part of this algorithm is novel clustering algorithms that are sets the names of factors of web-service procedures into semantically important ideas. These ideas are leveraged to find similarity of inputs (or 


\section{International Journal of Science and Research (IJSR) \\ ISSN (Online): 2319-7064}

Index Copernicus Value (2013): 6.14 | Impact Factor (2014): 5.611

outcome) of web-service procedures. The result of this paper considerably increases the precision and recalls matched with existing methods. To design Woogle is to contain automatic web-service calls this is disadvantage of this system. Woogle is showing to fill in the input factors and raise the procedures automatically for the customer after determining the good procedures.

\section{Issues}

\subsection{Cold-start}

Its difficult to give recommendations to new users as his taste is unknown to the system because his profile is almost empty and he has not rated any items. This is called the cold start problem. In some recommender systems this problem is has been solved by survey when creating a profile. Items can also have a cold-start when they are new in the system and there is no rating for them. Both of these problems can be also solved with hybrid approaches.

\subsection{Scalability}

In the entire environment where recommendation plays a important role, involves various products or services. Hence computational complexity of recommending product or service is proportional to the number of products or services and number of active users ${ }^{\text {ec }}$. However this problem can be solved by employing effective recommender algorithm.

\subsection{Trust}

The voices of people with a short history may not be considered as relevant as the voices of those who have large history in their profiles. The issue of trust is evaluating a certain customer. The problem could be solved by distribution of priorities to the users.

\subsection{Sparsity}

In online shops that have a huge amount of users and items. there are few users that have not rated much items. Using collaborative and other techniques recommender systems generally create neighborhoods of users using their profiles. If a user has evaluated just few items then its pretty difficult to determine his taste and user could be related to the inappropriate neighborhood. Sparsity is the problem of lack of information.

\subsection{Privacy}

Privacy has been the most important problem. In order to receive the most accurate and correct recommendation, the system must consider the large amount of information about the user, including data about the location of a particular user and demographic data. the question of security, reliability and confidentiality of the given information arises. Many systems offer effective protection of privacy of the users by utilizing specialized algorithms.

\subsection{Gray-sheep problem}

Competitive service provider might provide poor ratings to its competitive services thereby decreasing its chance of being recommended. Similarly, competitive service provider might provide good ratings to its own services thereby increasing its chance of being recommended. Even some users $^{\text {ee }}$ acting as malicious users ${ }^{\text {ee }}$ might provide inappropriate ratings to products or services. For new users"e, user profile will be initially created with no rating of targeted users ${ }^{\text {ee }}$ by other users ${ }^{e e}$. This approach is similar for items or services too.

\subsection{Ramp-up problem}

It is a Similar to cold-start problem.

\subsection{Synonym problem}

Most product or service either similar or same available seems to have different entries as services. The problem of Collaborative Filtering is that it doesn't handle this type of association.

\subsection{Shilling attack}

Competitive service provider might provide poor ratings to targeted services or good ratings to its own services, similar to gray sheep problem.

\section{Conclusion}

In This paper, we presented various type of recommendation system implemented based upon different attributes. We compared these different systems to derive the areas in which a particular strategy can be applied. We also have discussed the pros and cons of different Techniques. then we presented the web service recommendation related research. We have also uncovered issues that are open to many further improvements, In future various other attributes and techniques can be developed and evaluated for efficient implementation of recommendation systems.

\section{References}

[1] J.S.Breese, D. Heckerman, and C. Kadie, "Empirical Analysis of Predictive Algorithms for Collaborative Filtering," Proc. 14th Conf, Uncertainty in Artificial Intelligence (UAI '98), pp. 43-52, 1998.

[2] Atisha Sachan, "A Survey on Recommender Systems based on Collaborative Filtering Technique"l, International journal of Innovations in Engineering and technology (IJIET), ISSN. 2319-1058, Volume 2 Issue 2, April 2013.

[3] Urmela, Dr. K. Suresh Joseph, K. Vaitheki "A Survey on Web Service Mining by Collaborative Filtering and QoS" International Journal of Recent Development in Engineering and Technology (IJRDET), ISSN 23476435 Volume 3, Issue 3, September 2014. 
[4] I. H. and Frank I. Data Mining, Morgan Kaufman Publishers, San Francisco, 2000.

[5] A.Elgohary, H. Nomir, I. Sabek, M. Samir, M. Badawy, and N.A.Yousri, "Wiki-rec: A semantic-based recommendation system usingwikipedia as an ontology," in Intelligent Systems Design and Applications(ISDA), 2010 10th International Conference on,2010.

[6] K.O. et al."Context-aware svstem for context-dependent information recommendation".In International Conference On Mobile Data Management, 2006.

[7] Tejal Arekar, Mrs. R.S. Sonar,Dr. N. J. Uke "A Survey on Recommendation System" International Journal of Innovative Research in Advanced Engineering (IJIRAE) ISSN: 2349-2163Volume 2 Issue 1, January 2015.

[8] W. Lo, J. Yin, S. Deng, Y. Li, and Z. Wu, "An extended matrix factorization approach for qos prediction in service selection," in Proc. of the 9th IEEE International Conference on Services Computing (SCC), , pp. 162, 2012.

[9] Z. Maamar, S.K. Mostefaoui, and Q.H. Mahmoud, "Context for Personalized Web Services," Proc. 38th Ann. Hawaii Int'1 Conf., pp. 166b, 2005.

[10] X. Chen, Z. Zheng, X. Liu, Z. Huang, and H. Sun, „Personalized QoS-Aware Web Service Recommendation and Visualization, ${ }^{\text {ece }}$ IEEE Trans. Serv. Computing., vol. 6, no. 1, pp. 35-47, 2013.

[11] J. Yin, S. Deng, Y. Li, and Z. Wu, „,Collaborative Web Service QoS Prediction with Location-Based Regularization, ${ }^{\text {,ece }}$ in Proceedings of the 19th International Conference Web Services (ICWS 12 ), pp. 464-471, 2012.

[12] J. Zhu, Y. Kang, Z. Zheng, and M.R. Lyu, „A Clustering-Based QoS Prediction Approach for Web Service Recommendation, ${ }^{\text {eee }}$ in Proc. 15th IEEE Int' 1 Symp. Obj./Compon./Serv.- Oriented Real-Time Distrib. Comput. Workshops, pp. 93-98, Apr. 2012.

[13] G. Kang, J. Liu, M. Tang, X. Liu, B. Cao, and Y. Xu, „AWSR: Active Web Service Recommendation Based on Usage History" nin Proc. IEEE 19th ICWS, pp. 186193., 2012.

[14]Z. Zheng, H. Ma, M.R. Lyu, and I. King, ,,QoS-Aware Web Service Recommendation by Collaborative Filtering, ${ }^{\text {eec }}$ IEEE Trans. Serv. Comput., vol. 4, no. 2, pp. 140-152, Apr.June 2011. 